Supplement of Biogeosciences, 14, 5039-5051, 2017

https://doi.org/10.5194/bg-14-5039-2017-supplement

(C) Author(s) 2017. This work is distributed under

the Creative Commons Attribution 4.0 License.

(c) (1)

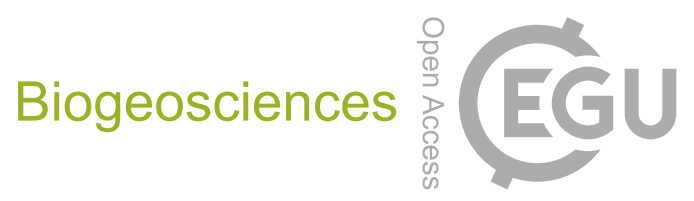

Supplement of

\title{
New molecular evidence for surface and sub-surface soil erosion controls on the composition of stream DOM during storm events
}

Marie Denis et al.

Correspondence to: Marie Denis (mariedenis57@hotmail.fr)

The copyright of individual parts of the supplement might differ from the CC BY 4.0 License. 
Table S1: List of the analysed compounds and their appropriate $\mathrm{m} / \mathrm{z}$ ratio and mass spectra factor (MSF). * indicate compounds retained for PCA analysis

\begin{tabular}{|c|c|c|}
\hline Compound name & $\mathrm{m} / \mathrm{z}$ & MSF \\
\hline \multicolumn{3}{|l|}{ Lignin phenols } \\
\hline *3,4-dimethoxyacetophenone (acetovanillone) & 165 & 2,8 \\
\hline * 3,4-dimethoxybenzaldehyde (vanillin) & 166 & 4,2 \\
\hline * 3,4-dimethoxybenzoic acid, methyl ester (vanillic acid) & 196 & 5,6 \\
\hline * 3,4,5-trimethoxyacetophenone (acetosyringone) & 195 & 4,8 \\
\hline * 3,4,5-trimethoxybenzaldehyde (syringaldehyde) & 196 & 6,7 \\
\hline * 3,4,5-trimethoxybenzoic acid, methyl ester (syringic acid) & 226 & 5,3 \\
\hline * 3-(4-methoxyphenyl)-prop-2-enoic acid, methyl ester (p-coumaric acid) & 192 & 6,7 \\
\hline * 3-(3,4-dimethoxyphenyl)-prop-2-enoic acid, methyl ester (ferulic acid) & 222 & 3,7 \\
\hline * 4-methyl-1,2-dimethoxybenzène & 152 & 5,7 \\
\hline * 1,2,4-trimethoxybenzène & 168 & 4,3 \\
\hline * 1,3,5-trimethoxybenzène & 168 & 3,0 \\
\hline * 3-methoxybenzoic acid methylester & 135 & 3,9 \\
\hline * 4-methoxybenzoic acid methylester & 135 & 2,8 \\
\hline 1,2,3,4-tetramethylbenzène & 198 & 3,5 \\
\hline * 1,2,3,5-tetramethylbenzène & 198 & 13,5 \\
\hline * cis-1,2-Dimethoxy-4-(2-methoxyethenyl)benzene & 194 & 5,1 \\
\hline * trans-1,2-Dimethoxy-4-(2-methoxyethenyl)benzene & 194 & 5,1 \\
\hline 1,2-Dimethoxy-4-(1-methoxy-1-propenyl)benzene & 208 & 6,2 \\
\hline cis-1,2-Dimethoxy-4-(2-methoxy-1-propenyl)benzene & 208 & 5,7 \\
\hline trans-1,2-Dimethoxy-4-(2-methoxy-1-propenyl)benzene & 208 & 5,7 \\
\hline trans-1,2-Dimethoxy-4-(3-methoxy-1-propenyl)benzene & 208 & 5,6 \\
\hline * cis-1,2,3-Trimethoxy-5-(2-methoxyethenyl)benzene & 224 & 7,0 \\
\hline trans-1,2,3-Trimethoxy-5-(2-methoxyethenyl)benzene & 224 & 7,0 \\
\hline * erythro-1,2-dimethoxy-4-(1,2,3-trimethoxypropyl)benzene & 181 & 3,6 \\
\hline threo-1,2-dimethoxy-4-(1,2,3-trimethoxypropyl)benzene & 181 & 3,6 \\
\hline cis-1,2,3-Trimethoxy-5-(2-methoxy-1-propenyl)benzene & 238 & 7,0 \\
\hline trans-1,2,3-Trimethoxy-5-(2-methoxy-1-propenyl)benzene & 238 & 7,0 \\
\hline cis-1,2-Dimethoxy-4-(2,3-dimethoxy-1-propenyl)benzene & 238 & 8,5 \\
\hline trans-1,2-Dimethoxy-4-(2,3-dimethoxy-1-propenyl)benzene & 238 & 8,5 \\
\hline * erythro-1,2,3-Trimethoxy-5-(1,2,3-trimethoxypropyl)benzene & 211 & 2,1 \\
\hline threo-1,2,3-Trimethoxy-5-(1,2,3-trimethoxypropyl)benzene & 211 & 2,1 \\
\hline benzoic acid methyl ester & 105 & 2,9 \\
\hline \multicolumn{3}{|l|}{ Carbohydrates } \\
\hline * xylose (C5) & 129 & 4,0 \\
\hline * rhamnose (deoxyC6) & 129 & 4,0 \\
\hline * fucose (deoxy C6) & 129 & 4,0 \\
\hline * glucose $(\mathrm{C} 6)$ & 129 & 4,0 \\
\hline * galactose (C6) & 129 & 4,0 \\
\hline
\end{tabular}


Table S1 continued

Fatty acids

* $\mathrm{C}_{12: 0}$

$74 \quad 3,0$

* $\mathrm{C}_{13: 0}$

$74 \quad 4,1$

* brC $_{14: 0}$

$74 \quad 3,1$

* $\mathrm{C}_{14: 0}$

$74 \quad 3,1$

* isoC 15 :0

$74 \quad 3,3$

* anteC $15: 0$

$74 \quad 3,3$

* $\mathrm{C}_{15: 0}$

$74 \quad 3,3$

* brC $_{16: 0}$

$74 \quad 4,9$

* $\mathrm{C}_{16: 1}$

* $\mathrm{C}_{16: 0}$

* isoC $\mathrm{C}_{17: 0}$

* anteC $17: 0$

* $\mathrm{C}_{17: 0}$

* $\mathrm{C}_{18: 1}$

* $\mathrm{C}_{18: 0}$

*

* $\mathrm{C}_{19: 0}$

* $\alpha, \oplus$ diacid $\mathrm{C}_{16: 0}$

$\mathrm{C}_{20: 0}$

* $₫-O H ~ C 18: 0$

$\mathrm{C}_{21: 0}$

* $\alpha, \infty$ diacid $\mathrm{C}_{18: 0}$

$\mathrm{C}_{22: 0}$

*

$\mathrm{C}_{23: 0}$

* $\quad \alpha, \infty$ diacid $\mathrm{C}_{20: 0}$

*

* $\mathrm{C}_{25: 0}$

$74 \quad 13,5$

* $\alpha, \infty$ diacid $\mathrm{C}_{22: 0}$

* $\mathrm{C}_{26: 0}$

* $\omega-\mathrm{OH} \mathrm{C} \mathrm{C}_{24: 0}$ 

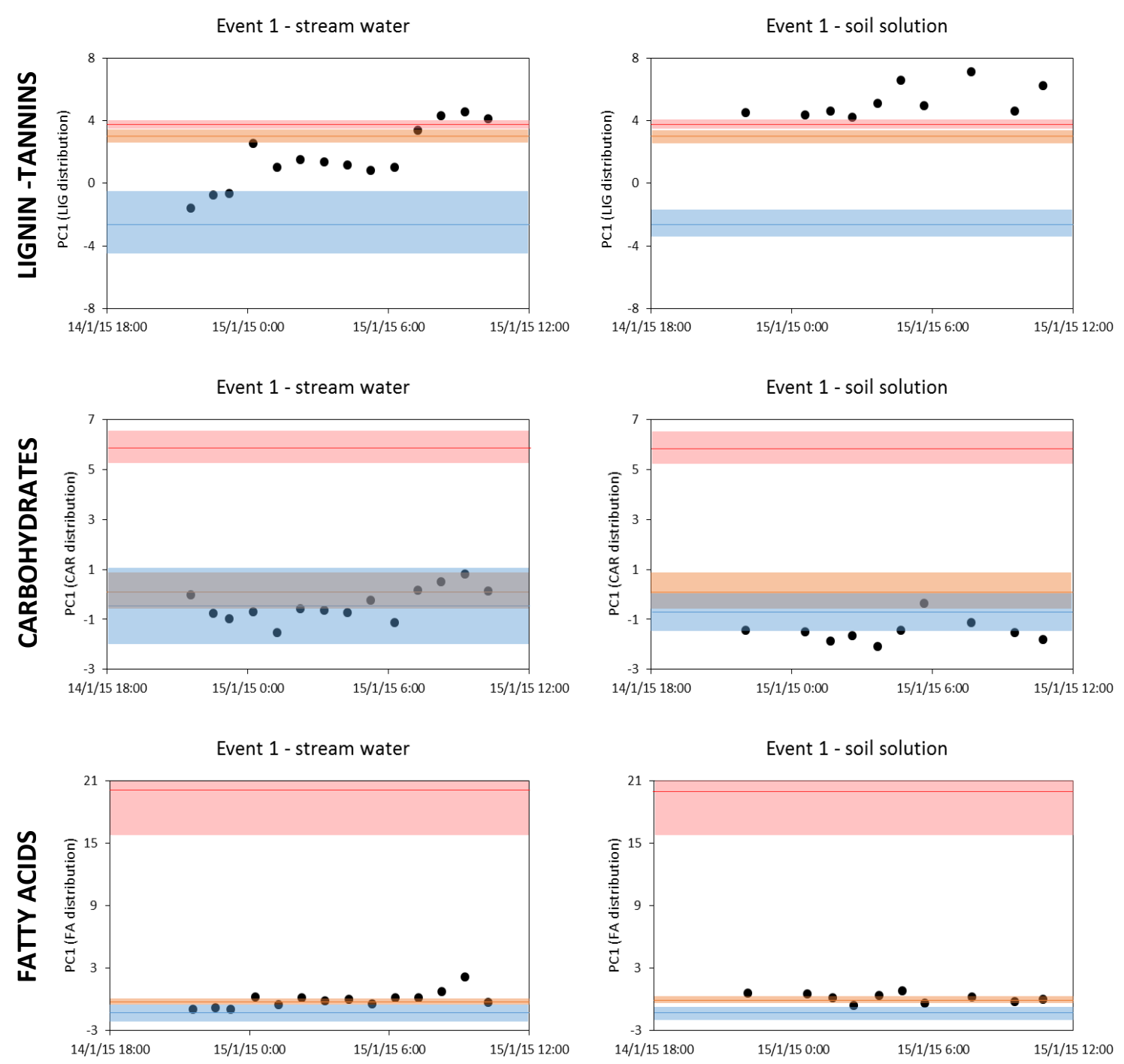

\section{Coordinate on PC1 axis (mean \pm standard deviation)}

Soil

Surface runoff

Coordinate on PC1 axis

Base flow

Figure S1: Evolution of LIG, CAR and FA distribution during the three events. Base flow, surface runoff and soil values are indicated by mean and standard deviation. 
Figure S1 (continued)
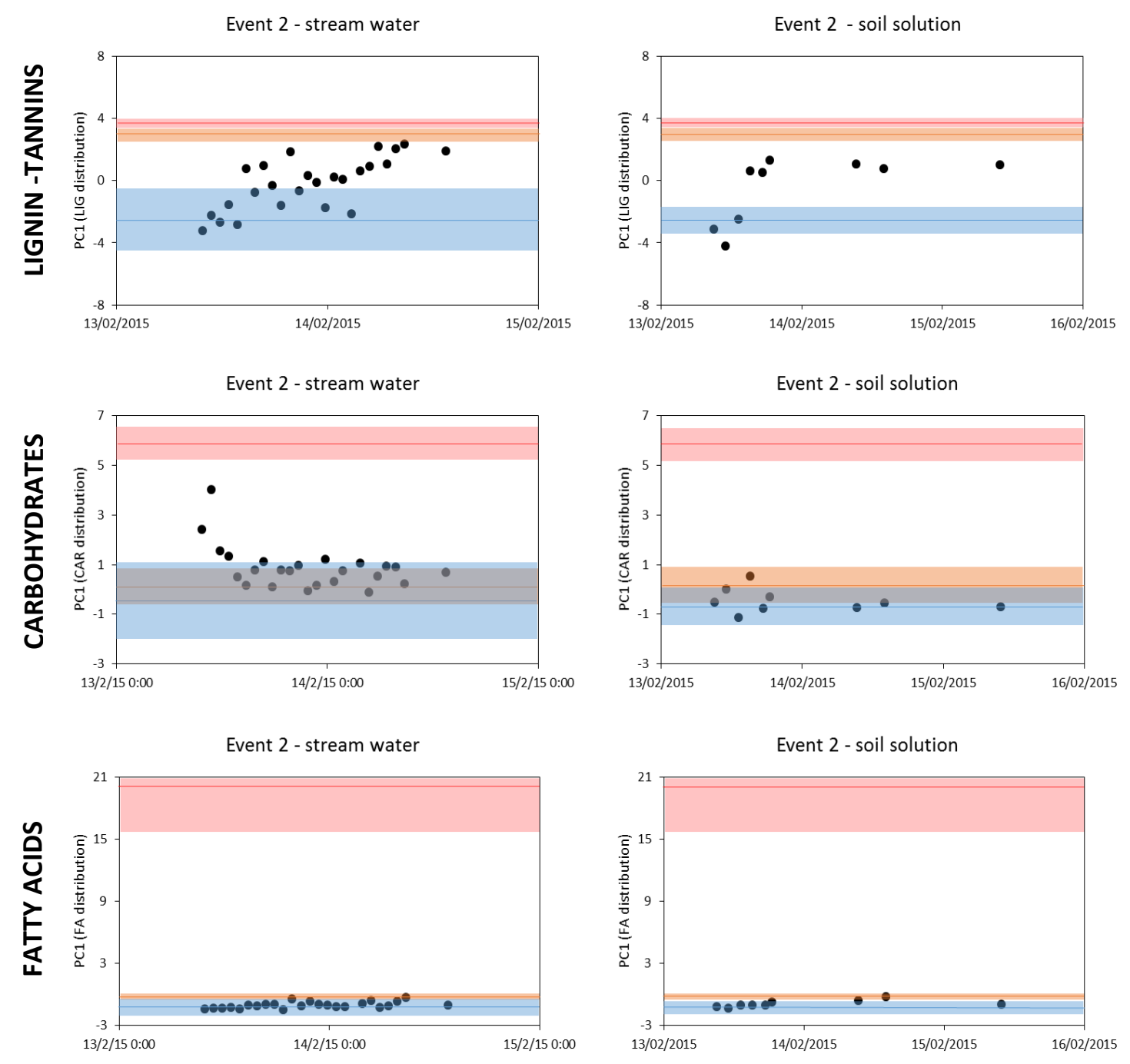

Figure S1 (continued) 

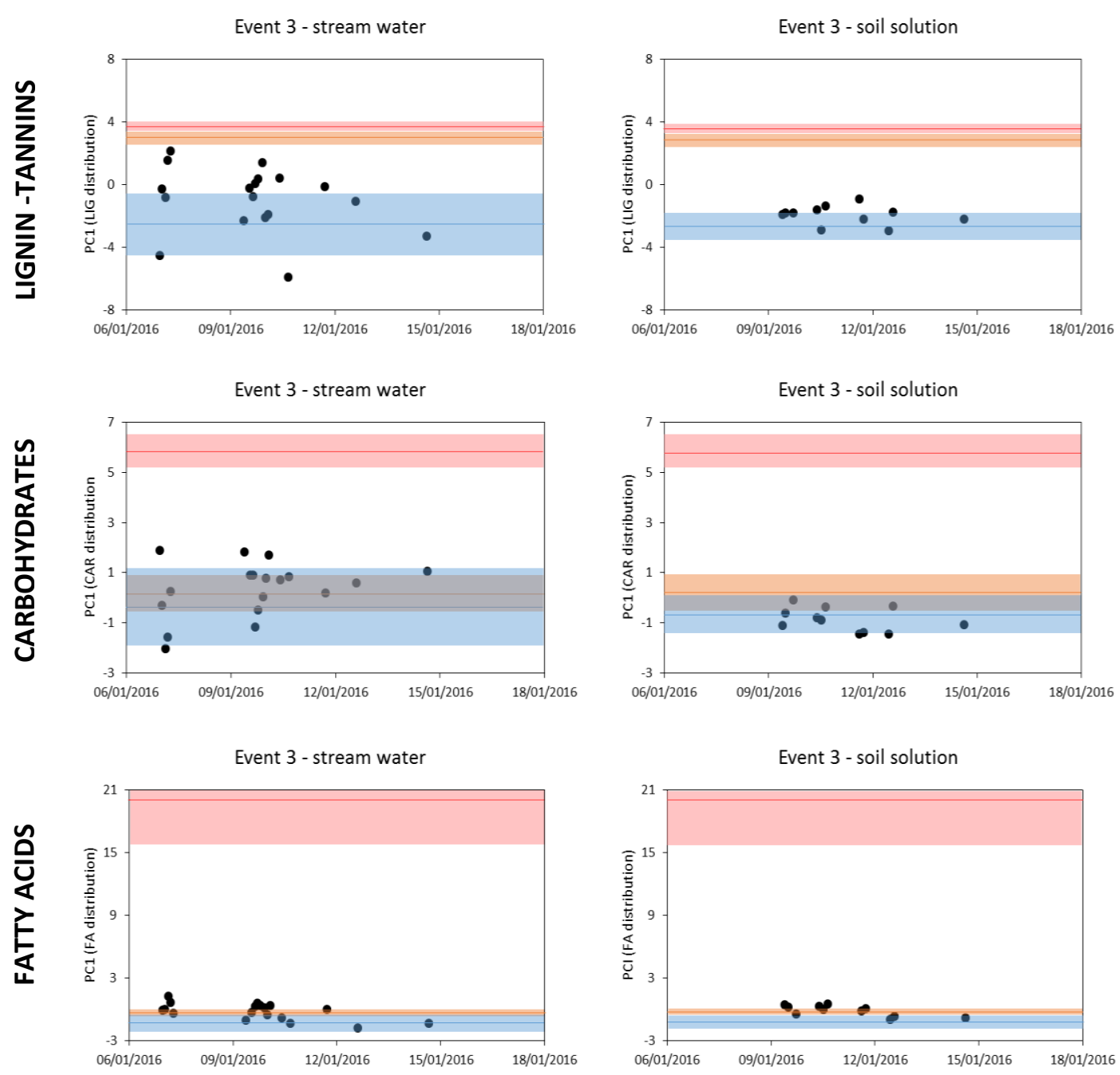

Figure S1 (continued) 


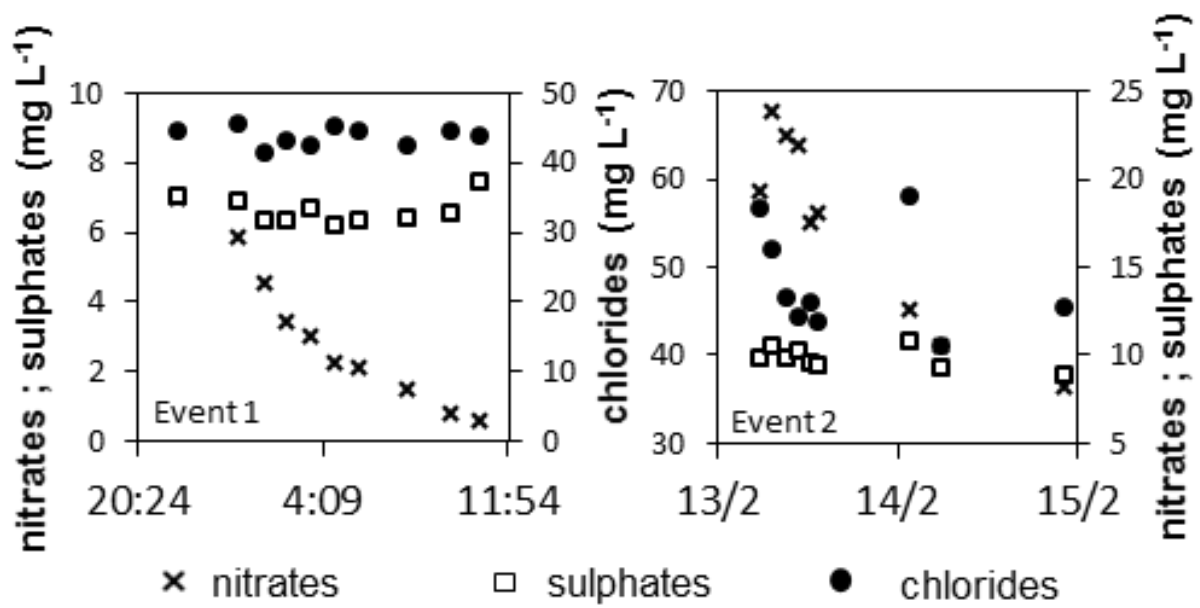

Figure S2: Concentration of nitrates, sulphates and chlorides in soil solutions during events 1 and 2.
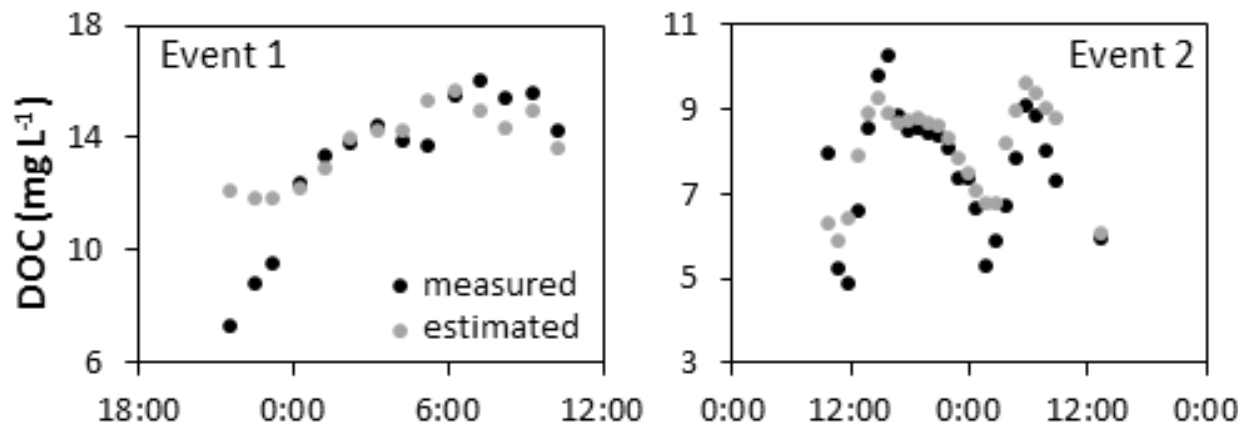

Figure S3: Comparison of DOC concentration measured at the outlet and DOC concentration estimated from deep groundwater, soil solution, surface runoff concentrations and their relative contribution to the discharge for events 1 and 2. 